\title{
Plasma-Mediated Immobilization of Antibody with PEG as Spacer for Enhanced Endothelial Cell Adhesion and Proliferation
}

\author{
Yuan Yuan, ${ }^{1,2,3}$ Jing Zhang, ${ }^{1,2,3}$ Min Yin, ${ }^{2,3}$ and Changsheng Liu, ${ }^{1,2,3}$ \\ ${ }^{1}$ The State Key Laboratory of Bioreactor Engineering, East China University of Science and Technology, Shanghai 200237, China \\ ${ }^{2}$ Key Laboratory for Ultrafine Materials of Ministry of Education and Engineering, East China University of Science and Technology, \\ Shanghai 200237, China \\ ${ }^{3}$ Research Center for Biomedical Materials of Ministry of Education, East China University of Science and Technology, \\ Shanghai 200237, China
}

Correspondence should be addressed to Yuan Yuan; yyuan@ecust.edu.cn and Changsheng Liu; csliu@sh163.net

Received 27 December 2013; Accepted 16 February 2014; Published 10 April 2014

Academic Editor: Traian V. Chirila

Copyright (C) 2014 Yuan Yuan et al. This is an open access article distributed under the Creative Commons Attribution License, which permits unrestricted use, distribution, and reproduction in any medium, provided the original work is properly cited.

\begin{abstract}
Immobilization of anti-CD34 antibody is proven to be an effective strategy to accelerate reendothelialization and thereby lower the thrombosis of blood contacting grafts. To realize highly efficient immobilization of anti-CD34 antibody, an argon cold plasmamediated graft process was developed with PEG as spacer arm in this study. In this process, the 316L stainless steel (316LSS) model substrate was first coated with ethylene vinyl acetate copolymer (EVA) followed by argon plasma treatment and PEG400 modification (EVA-PEG). The EVA-PEG was further ignited by argon plasma and then the anti-CD34 antibody was immobilized. XPS measurement indicated the successful immobilization of the EVA and the anti-CD34 antibody molecules. Compared with the anti-CD34 antibody anchored without PEG, the immobilized EVA-PEG-anti-CD34 antibody exhibited better capturing efficiency (increase about 1-fold) of specific antigen. Consequently, the endothelial cell attachment (before $12 \mathrm{~h}$ ) and proliferation (1 4 days) were significantly improved. Further study showed that this EVA-PEG-anti-CD34 coating could reduce blood coagulation. Therefore, this cold plasma-mediated graft process with PEG spacer arm developed here is a promising strategy to immobilize antibody with higher bioactivity for rapid reendothelialization of the cardiovascular implants.
\end{abstract}

\section{Introduction}

Coronary artery stenting has made tremendous success in the treatment of coronary artery disease [1-3]. In particular, the drug-eluting stents, which incorporate drugs that can effectively reduce the proliferation and migration of smooth muscle, have been developed and several clinically proven products (Cypher, Taxus, etc.) are currently available on market $[2,3]$. Unfortunately, the Late Angiographic Stent Thrombosis (LAST) continues to be a major challenge, which often leads to the failure of stent implantation in clinic. It is well-accepted that the poor endothelium-paving, either due to the injury to and loss of the endothelium or the retard endothelialization in/after implantation, is the important contributor to this thrombosis [4-6]. Therefore, from this viewpoint, accelerating reendothelialization or relining at denudated regions after implantation should be the most effective strategy to improve the clinic patency rate, particularly in small and medium diameter vascular prostheses.

To achieve rapid reendothelialization, many different approaches have been developed in the past few years, including active endothelial cell coating $[7,8]$ and ECcompatible layer [9]. Among them, anti-CD34 antibodygrafted stent, which can specifically identify and capture the circulating endothelial progenitor cells (EPCs) and neighboring endothelial cells (ECs) via the unique high-affinity interactions between the coated antibodies and the specific antigen CD34 markers on cell surface, appears to be the most 
attractive [10-12]. As an antibody, both the quantity and the presentation of the anti-CD34 antibody on stent surface play critical roles in the ensuing bioactivity. Unfortunately, till now, most available approaches for antibody immobilization lie on either passive physical adsorption $[13,14]$ or covalent cross-linking reactions via the linkage moieties $[15,16]$. Consequently, these antibody molecules are closely anchored on the matrix surface, which not only affects the amount of antibody immobilized but also often leads to the reduction of the binding capacity of the antibody toward antigen. In addition, the process for antibody graft often impacts the bioactivity of antibody. Therefore, there is a great need to develop an alternative anti-CD34 antibody immobilization strategy to fabricate high-performance antibody-coated blood grafts.

Herein, we developed a cold plasma-mediated grafting process for the immobilization of anti-CD34 antibody on the blood-contact materials. Also, to extend the bioactive site away from the surface and thus improve the accessibility of the anchored antibody, PEG was used as a spacer arm. To achieve this aim, 316L stainless steel (316LSS), a commonly used intravascular prosthesis, was selected as model substrate, on which the ethylene vinyl acetate (EVA) copolymer, extensively used on biomedical implant due to good stability, elasticity, and biocompatibility $[17,18]$, was firstly coated and followed by PEG and anti-CD34 antibody. Prior to the PEG and antibody immobilization, the EVA and EVA-PEG surface were all treated by argon cold plasma. For comparison, the anti-CD34 was also directly grafted on EVA. The modified coating surfaces were analyzed by XPS and water contact angles. The amount of the antibody immobilized and the binding capacity of the antibody/antigen were assayed. And the "in vitro" response of endothelial cells and the platelet adhesion on various surfaces were investigated.

\section{Materials and Methods}

2.1. Materials. Biomedical 316LSS was purchased from Shanghai Biomedical Metal Company, China. EVA (28 wt \% of vinyl acetate) and PEG ( $\mathrm{Mw}=400)$ were obtained from Shanghai Chemical Engineering Research Institute (China) and Jinan Dai Gang Biological Technology Co. (Jinan, China). The antibody (monoclonal mouse anti-human CD34 antibody) and CD34 were purchased from Gene. Tech. Co., Ltd., China. The human umbilical cord veins' endothelial cells (HUVECs) were obtained from China Centre for Type Culture Collection. RPMI-1640 medium was purchased from Gibco Co. (USA). Fetal calf serum (FCS), L-glutamine, periodate sodium, borohydride sodium, and rhodamine were all purchased from Sigma-Aldrich (Sigma, St. Louis, MO, USA).

\subsection{Methods}

2.2.1. Preparation of EVA Coatings onto 316LSS. The biomedical 316LSS slides $\left(1 \times 1 \mathrm{~cm}^{2}\right)$ were polished and ultrasonically cleaned with isopropanol in advance. After that, 4\% EVA solution was spin-coated onto 316LSS slides and evaporated under vacuum at $60^{\circ} \mathrm{C}$ for $24 \mathrm{~h}$. Then, the coatings were kept under vacuum at room temperature for later application.

2.2.2. Immobilization of PEG as Spacer Arm. To generate appropriate active site, the EVA-coated 316LSS was pretreated with argon plasma. The plasma set-up, the same as described in [19], consists of a reactor system, gas flow system and an RF power source $(13.56 \mathrm{MHz})$ with a matching network (ModelSPII), shielding, and a vacuum system. The EVA-SS substrates were subjected to argon plasma $(20 \mathrm{~Pa}, 100 \mathrm{w})$ treatment for $1 \mathrm{~min}$ and then immediately soaked in 5\% PEG400 solution (w/w in 95\% ethanol) for $15 \mathrm{~min}$ at room temperature. After that, the samples were dried at $120^{\circ} \mathrm{C}$ for $60 \mathrm{~min}$. This EVA coating after PEG modification was named EVA-PEG.

2.2.3. Immobilization of Anti-CD34 with PEG as Spacer. The above EVA-PEG slides were further used for immobilization of anti-CD34 antibody. Briefly, the EVA-PEG slides were subjected to argon plasma $(20 \mathrm{~Pa}, 100 \mathrm{w})$ treatment for $1 \mathrm{~min}$ again. And then, the slides were immediately immersed in $20 \mu \mathrm{g} / \mathrm{mL}$ anti-CD34 solution and incubated for $2 \mathrm{~h}$ at $4^{\circ} \mathrm{C}$. To avoid the nonspecific adsorption, this EVA-PEG-antiCD34 slide was incubated with $1 \%$ BSA solution at room temperature for another $2 \mathrm{~h}$.

To immobilize anti-CD34 without PEG spacer, the above plasma-treated EVA was directly immersed into in $20 \mu \mathrm{g} / \mathrm{mL}$ anti-CD34 solution and incubated for $2 \mathrm{~h}$ at $4^{\circ} \mathrm{C}$. Likewise, the EVA-anti-CD34 slide was incubated with $1 \%$ bovine serum albumin (BSA) solution at room temperature for another $2 \mathrm{~h}$.

To prepare the EVA-PEG-anti-CD34 and EVA-anti-CD34 samples for cell culture, the anti-CD34 solution was sterilized by filtration $(0.22 \mu \mathrm{m}$ in diameter $)$. And the experiments were all carried out in sterilized cleaning room.

2.2.4. Surface Characterization. The chemical composition changes of the 316LSS-EVA before and after antibody immobilization were investigated by X-ray photoelectron spectroscopy (XPS, MICROLAB MKaII, VG, UK). X-rays were generated with an $\mathrm{Mg}$ anode at a power of $300 \mathrm{~W}$. A constant take-off angle of $90^{\circ}$ with respect to the sample surface was used. The base pressure in the analysis chamber was $1 \times$ $10^{-6} \mathrm{~Pa}$. The surface charge effect was neutralized by the flood gun. Wide scan spectra in the $1000.0 \mathrm{eV}$ binding energy range were recorded with pass energy of $50 \mathrm{eV}$ for all samples. Core level scans were taken for carbon at pass energy of $0.1 \mathrm{eV}$.

To explore the change of the surface hydrophilicity, the substrates were characterized by static water contact angle measurements using JC2000A system (Shanghai Zhongchen Digital Equipment Co., Ltd., China) equipped with Panasonic GL350 camera. For this purpose, water droplets each of approximately $10 \mu \mathrm{L}$ were mounted over five different places on the surface of the substrate. The mean of $\theta$ value was calculated as an average of the number of measures taken.

2.2.5. Binding Capacity of the Antibody/Antigen. The adsorption amount of anti-CD34 and the binding capacity of the anti-CD34 immobilized to CD34 with and without PEG were investigated and compared. Prior to adsorption, the anti-CD34 and the CD34 were first labeled with rhodamine 


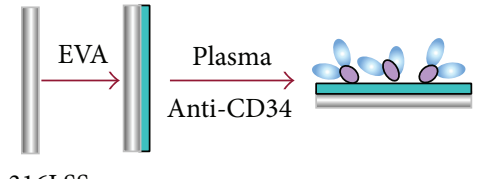

(a)

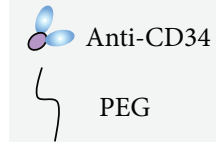

316LSS

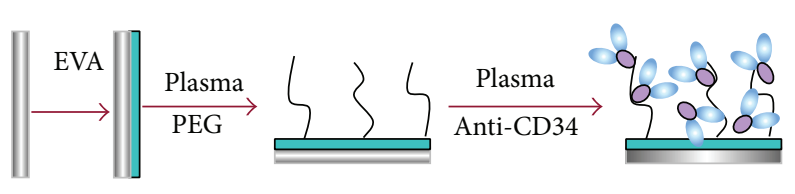

(b)

FIGURE 1: Schematic diagram of the immobilization of anti-CD34 by plasma process (a) without PEG spacer arm and (b) with PEG space.

according to the protocol of EZ-Label rhodamine Protein Labeling Kit. Briefly, BupH Borate Buffer Pack was reconstituted with $500 \mathrm{~mL}$ of ultrapure water and $100 \mu \mathrm{L}$ antiCD34/CD34 solution was dialysed into BupH Borate Buffer to a concentration of $1 \mathrm{mg} / \mathrm{mL}$. Rhodamine labeling reagent was completely dissolved in $100 \mu \mathrm{L}$ of dimethyl formamide (DMF). After that, $100 \mu \mathrm{L}$ anti-CD34/CD34 solution and an appropriate amount of rhodamine solution were mixed and incubated at room temperature for $1 \mathrm{~h}$. Finally, excess unbound fluorescent dye was removed by dialysis against $1 \mathrm{x}$ phosphate buffer solution (PBS).

To investigate the amount of anti-CD34 adsorbed with and without PEG, EVA and EVA-PEG slides after plasma pretreatment were directly incubated with the $15 \mu \mathrm{g} / \mathrm{mL}$ rhodamine-labeled anti-CD34 solution at $4^{\circ} \mathrm{C}$ for $24 \mathrm{~h}$. After being thoroughly rinsed with distilled water and blown dry, the fluorescent intensity after rhodamine-anti-CD34 adsorption was measured on Biochip reader (HD-2001A).

To assess the immunoreaction bioactivity, the EVA-antiCD34 and EVA-PEG-anti-CD34 slides were first blocked with $1 \% \mathrm{BSA}$ at room temperature for $2 \mathrm{~h}$ and then incubated with $100 \mu \mathrm{g} / \mathrm{mL}$ rhodamine-labeled CD34 solution at $4^{\circ} \mathrm{C}$ for another $2 \mathrm{~h}$. The fluorescent intensity was also measured on the aforementioned Biochip reader.

2.2.6. Cell Culture. The HUVECs were incubated in a culture medium consisting of $20 \%(\mathrm{v} / \mathrm{v})$ FCS and $80 \%$ RPMI1640 supplemented with $100 \mathrm{U} / \mathrm{mL}$ penicillin and $100 \mathrm{mg} / \mathrm{mL}$ streptomycin in humidified air containing $5 \% \mathrm{CO}_{2}$ at $37^{\circ} \mathrm{C}$.

To investigate and compare the cell attachment in the initial period, the substrates (including EVA, EVA-PEG, EVAanti-CD34, and EVA-PEG-anti-CD34) were kept in 24-well plates and the HUVECs were seeded at a cell density of $5 \times$ $10^{4}$ cells per well. After $4 \mathrm{~h}$, the cells were digested with $0.25 \%$ trypsin solution and the number of cells attached on different surfaces was determined by hemocytometer counting.

For cell growth studies, the samples were prepared with the same procedure. Cells were seeded at a density of $2.5 \times$ $10^{4}$ cells per well. The culture media were replenished every second day. After 1, 2,3, and 4 days, the cells grown on samples were treated and the number of cells grown on the samples was determined by the same method as the above-mentioned one.

The morphologies of the cells on different substrates after seeding for $12 \mathrm{~h}$ were observed by confocal laser scanning microscopy. The samples were washed with $0.1 \mathrm{M}$ cacodylate buffer ( $\mathrm{pH}$ 7.4) to remove the unattached cells. The HUVECs were fixed with $2.5 \%$ glutaraldehyde solution for $1 \mathrm{~h}$ at $4^{\circ} \mathrm{C}$. Then, the samples were labeled by FITC fluorescence according to the labeling kit protocol (Pierce Biotechnology, Rockford, IL). The morphologies of the cells were observed under a confocal laser scanning microscopy (CLSM, MRC1024, Bio-Rad, USA).

2.2.7. In Vitro Platelet Studies. For platelet adhesion studies, fresh human blood anticoagulated with acid citrate dextrose was centrifuged at $1000 \mathrm{rpm}$ for $10 \mathrm{~min}$ to obtain platelet-rich plasma (PRP). The samples were submerged with PRP for $1 \mathrm{~h}$ at $37^{\circ} \mathrm{C}$ in an incubator. After washing gently with PBS to remove nonadhering platelets, fixing was done with $2.5 \%$ buffered glutaraldehyde solution for $1 \mathrm{~h}$ at $37^{\circ} \mathrm{C}$. Specimens were then dehydrated with $50,70,95$, and 100 dilutions of ethanol and water and dried. All specimens were coated with a gold layer and observed with SEM (JSM-6360LV, JEOL, Japan).

2.2.8. Statistical Analysis. Statistical analysis was performed with one-way analysis of variance (ANOVA). Differences were considered to be significant at a level of $P<0.05$.

\section{Results}

3.1. Immobilization and Characterization of the Immobilized Anti-CD34. In this study, to maintain the bioactivity of anti-CD34 antibody immobilized on 316LSS, EVA was first coated and followed by PEG as spacer arm. To ensure the efficient immobilization of EVA and antibody, the EVA and EVA-PEG surface were all treated by argon cold plasma in advance (Figure 1(a)). For comparison, the anti-CD34 was also directly grafted on EVA (Figure 1(b)).

To avoid the steric hindrance of the antibody attached, the concentration of the anti-CD34 was preliminarily optimized and fixed at $20 \mu \mathrm{g} / \mathrm{mL}$. The successful immobilizations of PEG400 and anti-CD34 onto the substrate by cold plasma-induced graft process were directly confirmed by the chemical composition determined by XPS analyses. Figure 2 showed the survey XPS spectra of different coating surfaces, where (a), (b), and (c) correspond to EVA, EVAPEG, and EVA-PEG-anti-CD34, respectively. It can be seen that EVA before and after PEG grafting all exhibited two peaks corresponding to $\mathrm{C} 1 s(285 \mathrm{eV})$ and $\mathrm{O} 1 s(532 \mathrm{eV})$, while, after being further coated with anti-CD34, an additional peak corresponding to N1s (binding energy, $400 \mathrm{eV}$ ) emerged, indicating that anti-CD34 was grafted on the substrates. The surface chemical compositions of different coatings were also analyzed. The oxygen content of EVA was increased from 


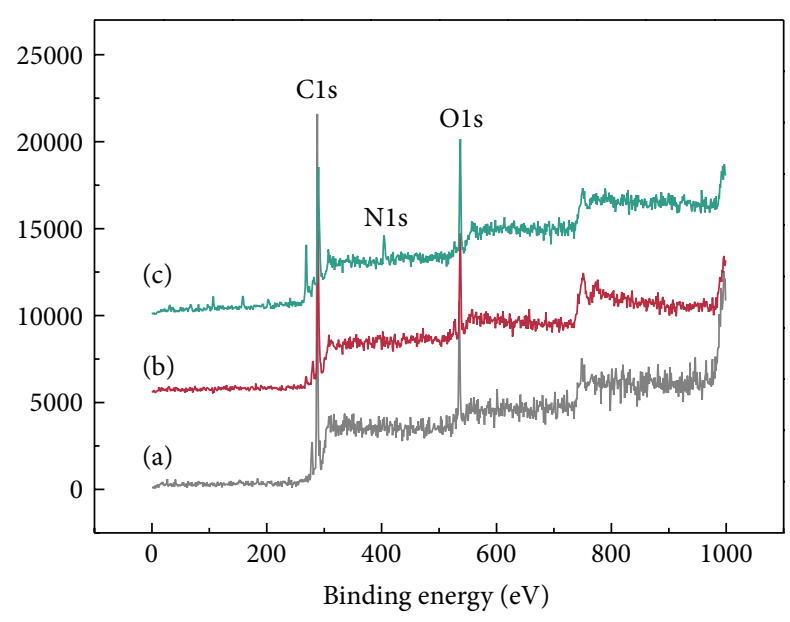

FIgUre 2: Survey XPS spectra of (a) EVA, (b) EVA-PEG, and (c) EVA-PEG-anti-CD34.

TABLE 1: Water contact angles of different surfaces.

\begin{tabular}{lccc}
\hline Samples & EVA & EVA-PEG & EVA-PEG-anti-CD34 \\
\hline Contact angle $\left(^{\circ}\right)$ & $73.2^{\circ} \pm 2.0$ & $55.1^{\circ} \pm 3.6$ & $25.9^{\circ} \pm 1.9$ \\
\hline
\end{tabular}

29.3\% to $38.63 \%$ after PEG grafting, resulting from the high oxygen content of PEG, whereas, after being further treated with anti-CD34, the oxygen content was slightly decreased (37.12\%) and a low nitrogen component (4.25\%) was observed on the coating surface.

The high resolution C1s core level scan spectra of different coatings are shown in Figure 3. Deconvolution of the spectra was carried out using a Gaussian curve fitting technique, and the contributions were assumed due to carbon bonding according to other reports [20]. As shown in Figure 3, the C1s peak of EVA can be divided into three peaks corresponding to $\mathrm{C}-\mathrm{C}, \mathrm{C}-\mathrm{O}$, and $\mathrm{C}=\mathrm{O}$ at $284.5 \mathrm{eV}, 285.9 \mathrm{eV}$, and $288.8 \mathrm{eV}$, respectively. After PEG grafting, the peak at $284.5 \mathrm{eV}$ decreased, while the peaks at $285.9 \mathrm{eV}$ and $288.8 \mathrm{eV}$ increased. But, after anti-CD34 treatment, the peak at $285.9 \mathrm{eV}$ further increased and an additional peak corresponding to $\mathrm{CO}-\mathrm{NH}$ at $288 \mathrm{eV}$ appeared.

Table 1 presented the changes of water contact angles after various modifications. As shown, the original water contact angle of EVA coating was $73.2^{\circ}$. But, after further PEG and antibody immobilization, the contact angle decreased to about $55.1^{\circ}$ and $25.9^{\circ}$, respectively. These results confirmed the occurrence of grafting of PEG and antibody on the 316LSS-EVA surface again.

3.2. Binding Capacity of Antibody/Antigen on the EVA Surface. The adsorption of anti-CD34 and the subsequent CD34 recognition capacity of the anti-CD34 on different coatings were further examined. At the concentration of $20 \mu \mathrm{g} / \mathrm{mL}$ of anti-CD34, as shown in Figure 4, the amount of the IgG adsorbed on EVA-PEG (295 RUF) was obviously higher than that directly immobilized on the EVA surface (150 RUF). Furthermore, the amount of the recognized CD34 by antiCD34 immobilized on EVA-PEG increased almost 3-fold than EVA surface. We believed that the enhancement of antiCD34 attached can be attributable to the PEG effect, and the clear increase of affinity binding comes from a desirable orientation of anti-CD34 molecules on EVA-PEG surface.

\subsection{The Attachment and Growth of HUVECs on the Modified} Substrate. Figure 5 shows the attachment of HUVECs on 316LSS with different modifications after 3, 6, 9, and $12 \mathrm{~h}$ of culture, respectively. As shown, EVA-PEG had a slightly enhanced cell attachment compared with the EVA control at the specific time point. But the cell attachment was significantly improved after anti-CD34 immobilization. After $6 \mathrm{~h}$ incubation, compared with the control group (EVA), the number of the attached cells on the EVA-anti-CD34 and EVA-PEG-anti-CD34 were increased around $51 \%$ and $110 \%$, respectively. This tendency was strengthened after $12 \mathrm{~h}$ of culture. Compared to the EVA-anti-CD34, the endothelial cell attachment on EVA-PEG-anti-CD34 surface, after 3, 6, 9, and $12 \mathrm{~h}$ cultivation, increased about $1 \sim 2$-fold. These results suggested that the cell attachment was significantly stimulated by the presence of anti-CD34 antibody immobilized by this plasma-induced process, especially for that immobilized on the EVA-PEG surface.

Figure 6 shows the growth curves of HUVECs on 316LSS uncoated and coated with anti-CD34 over a period of 4 days. It was found that the cells significantly grew faster on the antiCD34-modified coating than those substrates without antiCD34 in the entire study period. The improvement is more obvious after 2 days. In particular, the EVA-PEG-anti-CD34 surface was more favorable for the cell proliferation than the EVA-anti-CD34 surface.

The morphology of HUVECs after $12 \mathrm{~h}$ of incubation on various surfaces was shown in Figure 7. The focal points of the HUVECs can be clearly seen and the cultured cells attached to the surface of the 316LSS before and after modification within $12 \mathrm{~h}$ cultivation. As shown, HUVECs on EVA and EVA-PEG surfaces did not spread well, showing a wound-shaped morphology. In contrast, on the anti-CD34containing 316LSS surfaces, not only the number of the HUVECs was greatly increased but also the morphology of the cells was more elongated than those cultured on EVA and EVA-PEG surfaces. Typical cobblestone-like appearance was seen and cells formed a confluent layer on the EVA-PEG-antiCD34 surface within the $12 \mathrm{~h}$ culture.

3.4. In Vitro Haemocompatibility of the Antibody Coating. Figure 8 shows the platelet adhesion on different coatings contacting with PRP for $60 \mathrm{~min}$. It can be observed that no obvious platelet adhesion and aggregation were observed on anti-CD34 immobilized surfaces, whereas a high platelet adhesion and aggregated cell platelets (about $4600 / \mathrm{mm}^{2}$ ) were clearly observed on the unmodified surface.

\section{Discussion}

It is well-accepted that rapid and successful reendothelialization or relining in vivo can largely prevent the thrombosis of the blood contacting implants, such as vascular grafts, stents, and heart valves [1-3]. Previously, our group and 


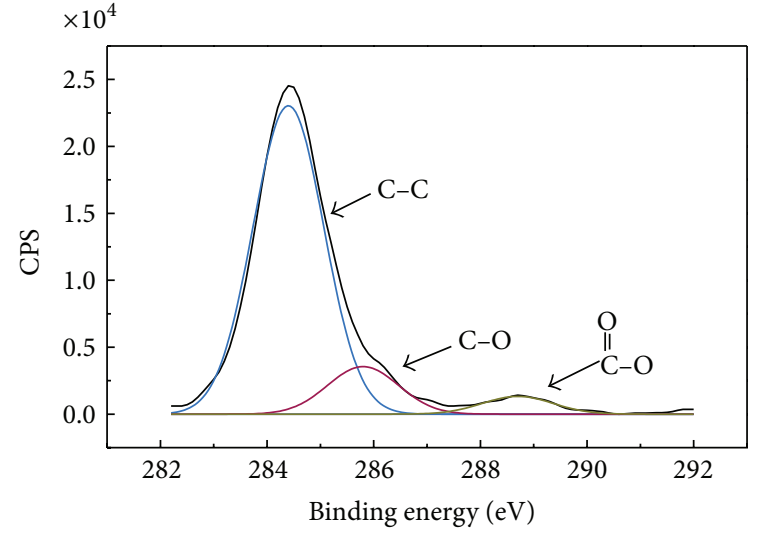

(a)

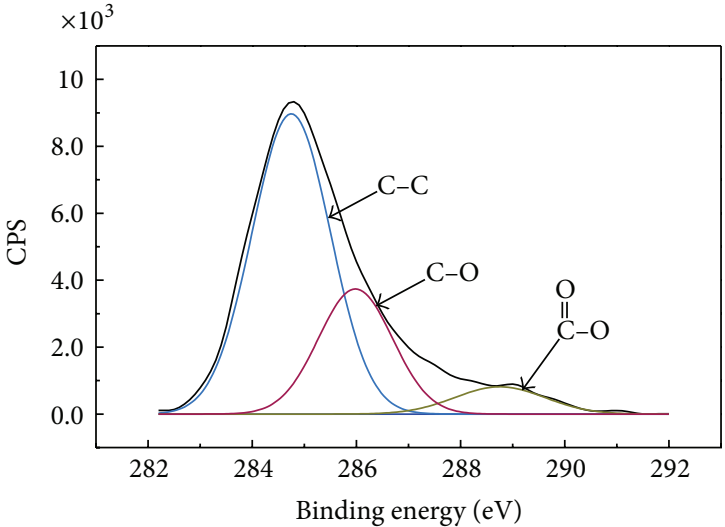

(b)

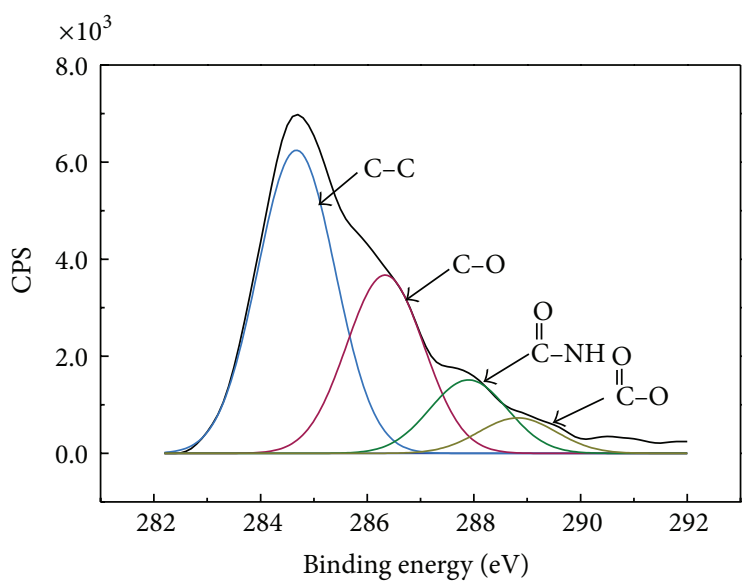

(c)

Figure 3: XPS carbon $1 s$ core level scan spectra of (a) EVA, (b) EVA-PEG, and (c) EVA-PEG-anti-CD34.

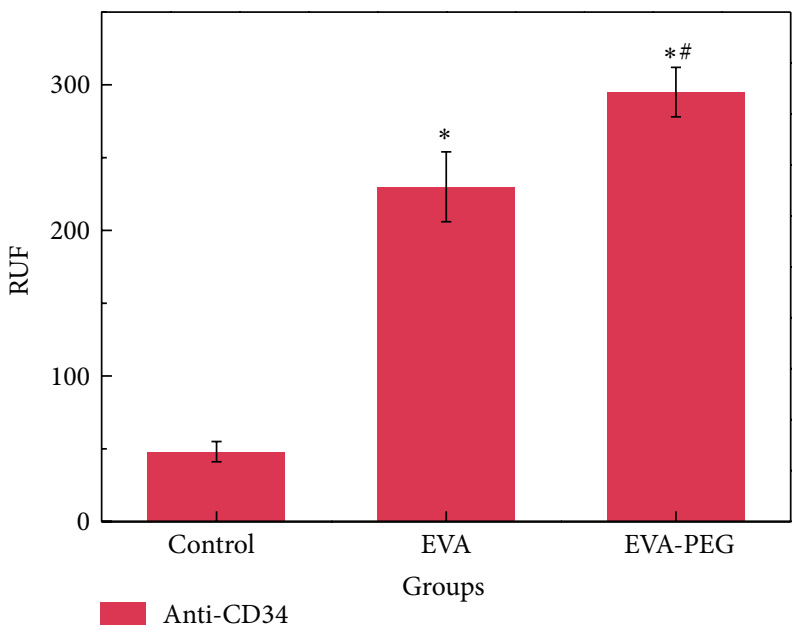

(a)

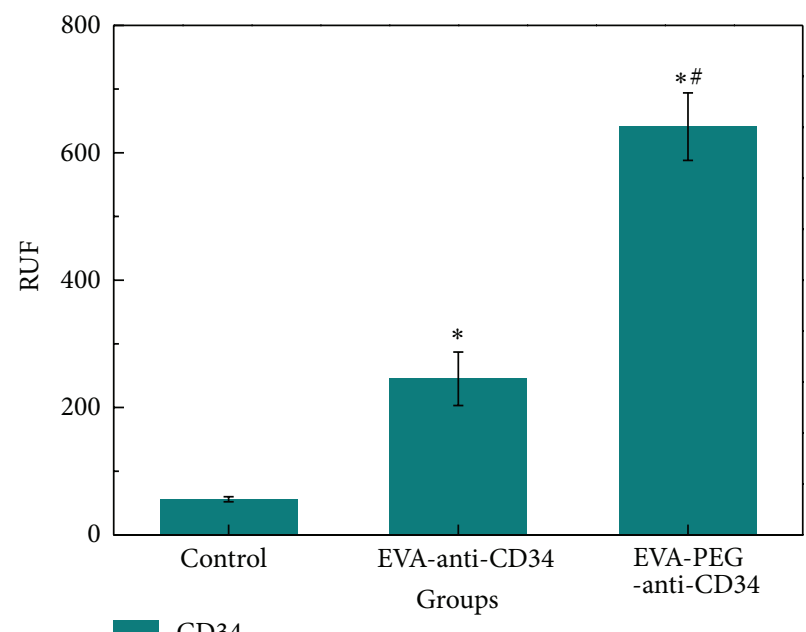

(b)

Figure 4: Comparison of the amount (a) and the bioactivity (b) of the anti-CD34 immobilized by different methods. The unlabeled antiCD34 and the oxidized anti-CD34 were all immobilized at the optimal conditions. And the CD34 was labeled with rhodamine and incubated with the antibody-immobilized samples at the following conditions: antibody concentration, $100 \mu \mathrm{g} / \mathrm{mL}$, for $2 \mathrm{~h}$ at $4^{\circ} \mathrm{C}\left({ }^{*} P<0.05\right.$ versus control group) ( ${ }^{*} P<0.05$ versus EVA-anti-CD34 group). 


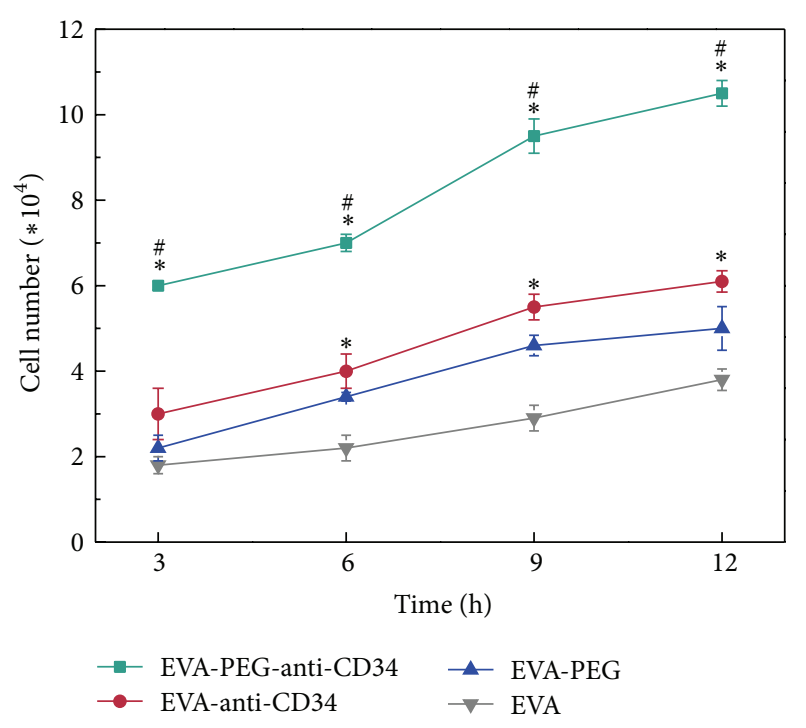

FIGURE 5: Comparison of the HUVECs attachment on the 316LSS with different modification methods after $12 \mathrm{~h}$ of culture. $\left({ }^{*} P<0.05\right.$ versus EVA group at corresponding time point.) $\left({ }^{\#} P<0.05\right.$ versus EVA-anti-CD34 group at corresponding time point.)

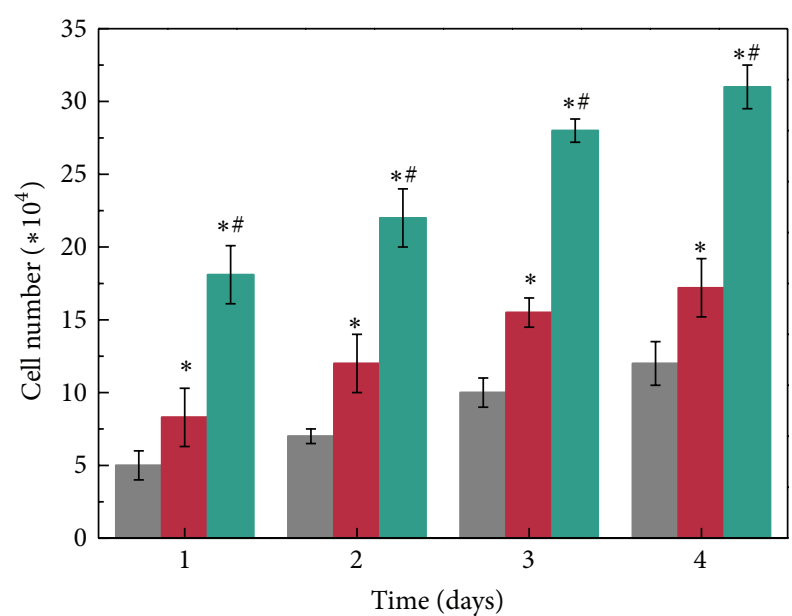

Control
EVA-anti-CD34

Figure 6: Growth of HUVECs on 316LSS with different modification methods after $1,2,3$, and 4 days of culture. $\left({ }^{*} P<0.05\right.$ versus control group at corresponding time point.) $\left({ }^{\#} P<0.05\right.$ versus EVAanti-CD34 group at corresponding time point.)

other researches have demonstrated that anti-CD34 immobilization was beneficial for enhanced HUVECs attachment and thus rapid reendothelialization [10]. In this work, we continued these studies to immobilize the anti-CD34 with PEG as spacer arm via cold plasma-induced graft process. With this cold plasma-induced graft method, the anti-CD34 can be attached on the substrate without any solvent and at low temperature. As a spacer arm, the PEG molecule can effectively extend the bioactive site away from the surface and introduce flexibility while reducing steric interferences between the active molecules and increasing the efficiency of the immobilized molecular [21, 22]. So, we hypothesized that, by combining the PEG-induced spacer arm effect and the cold plasma-induced graft process, the anti-CD34 molecule can be immobilized on the substrate with high bioactivity.

316LSS, which is commonly used as vascular stents, is distinguished by its excellent mechanic property and cost-effectiveness; however, relatively intrinsic inert property makes it very difficult to graft polymer onto its surface. Hence, an additional surface EVA copolymer coating was firstly spincoated to serve as an intermediate coating between 316LSS and antibody. According to the XPS spectra, the PEG and the anti-CD34 molecule can be successfully grafted on the 316LSS-EVA by cold plasma-induced graft technique process.

The binding of antibody and subsequent antigen recognition and cellular response determine the later antibodycoated grafts efficiency in clinic therapy. As expected, the results from Figure 4 revealed that, with the same substrate and same plasma treatment process, the anti-CD34 immobilized on the PEG surface are oriented more favorably for the capture of specific antigen molecules than that directly immobilized on the EVA. Additionally, compared with the EVA-anti-CD34, the EVA-PEG-anti-CD34 also facilitates the ensuing HUVECs cellular capture, attachment, and proliferation. This difference can be attributable to the spacer arm effect. It has been reported that the incorporation of a minimum spacer length, allowing the molecules to stay away from the solid surface and thus avoiding its denaturation, was imperative for bioactive molecule binding on the solid support material [22]. So, in this study, we only selected PEG400 as spacer arm based on the preliminary experiment. These results also confirmed that the anti-CD34 anchored with PEG 400 spacer arm via the cold-plasma grafting process exhibited desirable activity.

As a blood-contacting biomedical device, the stent surface should prevent the thrombus formation. In general, when materials contact with blood, proteins are first adsorbed instantaneously onto surfaces and deformed, and then platelets are adsorbed, activated, and aggregated so that platelets play a major role in the initial thrombus formation. Therefore, a study on platelet adhesion is the first step to evaluate the blood compatibility of materials. Figure 8 revealed that the platelet adhesion after anti-CD34 grafting was greatly reduced, indicating the improvement of blood compatibility. This good blood compatibility can be attributed to the high hydrophilicity, surface free energy arising from the $-\mathrm{OH}, \mathrm{C}=\mathrm{O}$, and $\mathrm{C}-\mathrm{O}$ functionalities. Also, it is clear that the PEG spacer arm can reduce the platelet adhesion. The dominating molecular mechanisms underlying protein resistance of grafted PEG are still not fully understood, but a brush-induced steric repulsion, preventing contact between proteins and the underlying surface, and the hydration shells around the PEG moieties, which energetically suppress the adsorption of proteins, are considered as the two dominating mechanisms [22].

In today's stenting implantation procedures, the thrombosis caused by the delayed endothelialization is one of the great challenges. Anti-CD34 immobilization has been considered as an effective strategy. The anti-CD34 immobilized on the 316LSS in this study exhibited higher antigen capture 


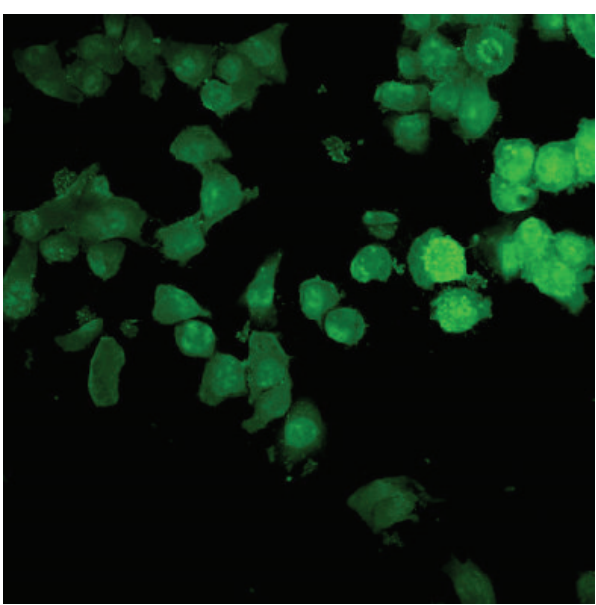

(a)

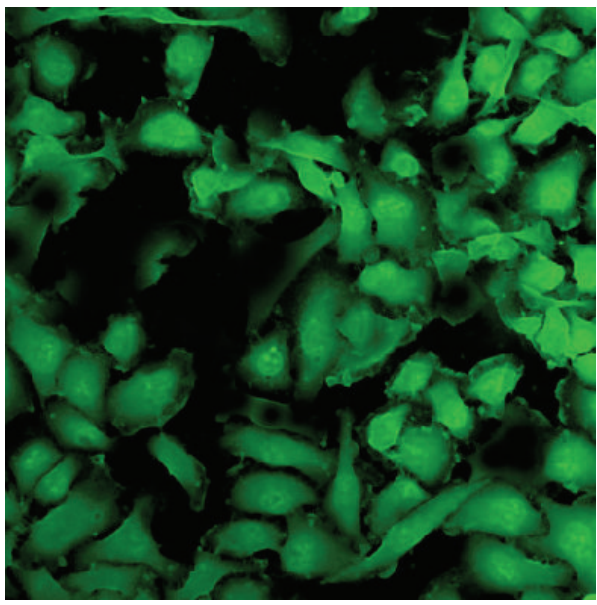

(c)

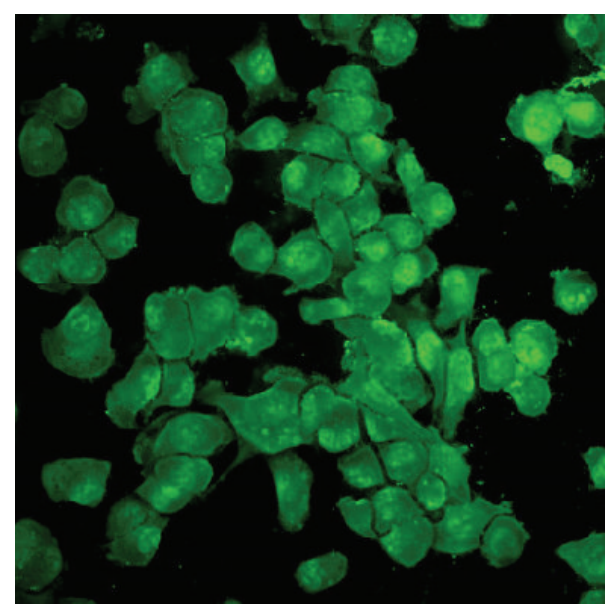

(b)

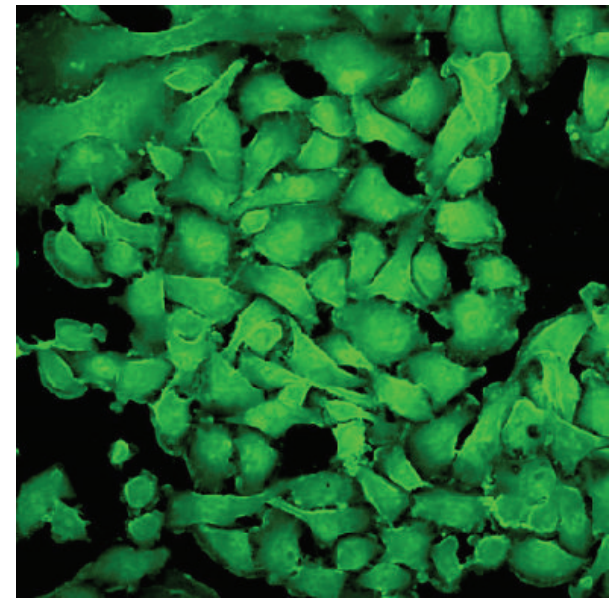

(d)

FIGURE 7: Morphologies of HUVECs $(\times 40)$ seeded on 316LSS with different modification methods: (a) EVA, (b) EVA-PEG, (c) EVA-antiCD34, and (d) EVA-PEG-anti-CD34.

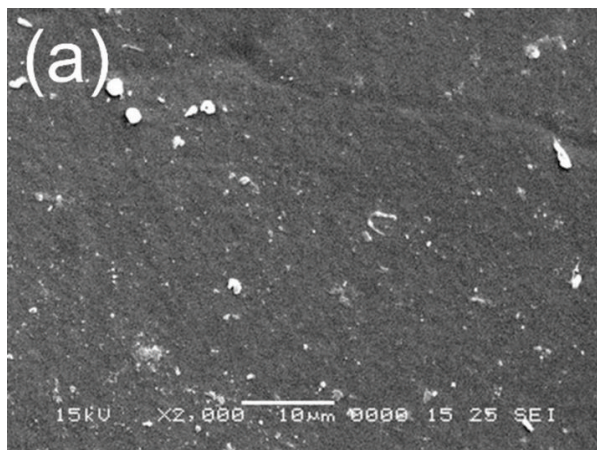

(a)

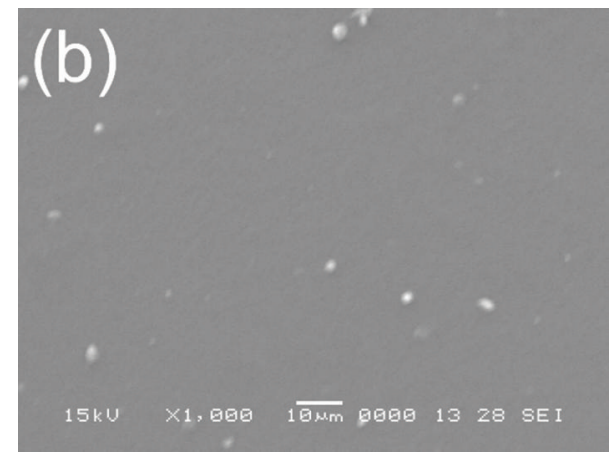

(b)

Figure 8: SEM images of the anti-CD34-coated 316LSS with PRP for $60 \mathrm{~min}$ : (a) 316LSS and (b) EVA-PEG-anti-CD34.

capacity and thus significantly enhanced the attachment and adhesion of HUVECs. Hence, it can be expected that the EVA-PEG-anti-CD34 could accelerate the reendothelialization and control the late thrombosis risk effectively.

\section{Conclusion}

In this work, anti-CD34 was immobilized onto EVA-coated 316LSS surface by cold plasma-induced grafting process with 
PEG as spacer arm. Due to the low temperature and less influence on antibody, together with the spacer arm effect of PEG, the antibody immobilized by this process proposed in this study exhibited higher antigen capture capacity and enhanced HUVECs cell attachment and proliferation. Meanwhile, the modified EVA-PEG-anti-CD34 coatings have favorable blood compatibility. It can be concluded that the EVA-PEG-antiCD34 coating immobilized through this developed cold plasma-induced grafting technique could simultaneously promote the formation of a confluent HUVECs layer and prevent thrombus formation, representing a promising strategy for rapid reendothelialization of intravascular stent devices.

\section{Conflict of Interests}

The authors declare that there is no conflict of interests regarding the publication of this paper.

\section{Acknowledgments}

The authors wish to express their gratitude for the financial support received from the National Basic Research Program of China (973 Program, no. 2012CB933600), the National Natural Science Foundation of China (nos. 31070850 and 31100679), and the Scientific Research Foundation for the Returned Overseas Chinese Scholars, State Education Ministry. This study was also supported by the Program for New Century Excellent Talents in University (no. NCET-11-0640).

\section{References}

[1] L. Mauri, W.-H. Hsieh, J. M. Massaro, K. K. L. Ho, R. D’Agostino, and D. E. Cutlip, "Stent thrombosis in randomized clinical trials of drug-eluting stents," New England Journal of Medicine, vol. 356, no. 10, pp. 1020-1029, 2007.

[2] M. Yin, Y. Yuan, C. S. Liu, and J. Wang, "Development of mussel adhesive polypeptide mimics coating for in-situ inducing reendothelialization of intravascular stent device," Biomaterials, vol. 30, no. 14, pp. 2764-2773, 2009.

[3] Mass High Tech, "Boston Scientific gets FDA OK for two Taxus stent uses," 2008, http://www.masshightech.com/stories/2008/ 09/22/daily47.

[4] S. Meng, Z. Liu, L. Shen et al., "The effect of a layer-bylayer chitosan-heparin coating on the endothelialization and coagulation properties of a coronary stent system," Biomaterials, vol. 30, no. 12, pp. 2276-2283, 2009.

[5] A. V. Finn, F. D. Kolodgie, J. Harnek et al., "Differential response of delayed healing and persistent inflammation at sites of overlapping sirolimus- or paclitaxel-eluting stents," Circulation, vol. 112, no. 2, pp. 270-278, 2005.

[6] Q. Lin, X. Ding, F. Qiu, X. Song, G. Fu, and J. Ji, "In situ endothelialization of intravascular stents coated with an antiCD34 antibody functionalized heparin-collagen multilayer," Biomaterials, vol. 31, no. 14, pp. 4017-4025, 2010.

[7] M. Avci-Adali, A. Paul, G. Ziemer, and H. P. Wendel, "New strategies for in vivo tissue engineering by mimicry of homing factors for self-endothelialisation of blood contacting materials," Biomaterials, vol. 29, no. 29, pp. 3936-3945, 2008.

[8] B. H. Walpoth and G. L. Bowlin, "The daunting quest for a small diameter vascular graft," Expert Review of Medical Devices, vol. 2, no. 6, pp. 647-651, 2005.
[9] M. Yin, Y. Yuan, C. Liu, and J. Wang, "Development of mussel adhesive polypeptide mimics coating for in-situ inducing reendothelialization of intravascular stent devices," Biomaterials, vol. 30, no. 14, pp. 2764-2773, 2009.

[10] Y. Yuan, M. Yin, J. Qian, and C. Liu, "Site-directed immobilization of antibodies onto blood contacting grafts for enhanced endothelial cell adhesion and proliferation," Soft Matter, vol. 7, no. 16, pp. 7207-7216, 2011.

[11] J. M. Lee, W. S. Choe, B. K. Kim et al., "Comparison of endothelialization and neointimal formation with stents coatedwith antibodies against CD34 and vascular endothelial-cadherin," Biomaterials, vol. 33, no. 35, pp. 8917-8927, 2012.

[12] F. Yang, S. C. Feng, X. J. Pang et al., "Combination coating of chitosan and anti-CD34 antibody applied on sirolimuseluting stents can promote endothelialization while reducing neointimal formation," BMC Cardiovascular Disorders, vol. 12, article 96, 2012.

[13] J. Aoki, P. W. Serruys, H. Van Beusekom et al., "Endothelial progenitor cell capture by stents coated with antibody against CD34: the HEALING-FIM (Healthy Endothelial Accelerated Lining Inhibits Neointimal Growth-First in Man) registry," Journal of the American College of Cardiology, vol. 45, no. 10, pp. 1574-1579, 2005.

[14] M. Yin, Y. Yuan, C. Liu, and J. Wang, “Combinatorial coating of adhesive polypeptide and anti-CD34 antibody for improved endothelial cell adhesion and proliferation," Journal of Materials Science: Materials in Medicine, vol. 20, no. 7, pp. 1513-1523, 2009.

[15] J. I. Rotmans, J. M. M. Heyligers, H. J. M. Verhagen et al., "In vivo cell seeding with anti-CD34 antibodies successfully accelerates endothelialization but stimulates intimal hyperplasia in porcine arteriovenous expanded polytetrafluoroethylene grafts," Circulation, vol. 112, no. 1, pp. 12-18, 2005.

[16] J. Chen, J. Cao, J. Wang et al., "Biofunctionalization of titanium with PEG and anti-CD34 for hemocompatibility and stimulated endothelialization," Journal of Colloid and Interface Science, vol. 368, no. 1, pp. 636-647, 2012.

[17] S. Kalachandra, L. Dongming, and S. Offenbacher, "Controlled drug release for oral condition by a novel device based on ethylene vinyl acetate (EVA) copolymer," Journal of Materials Science: Materials in Medicine, vol. 13, no. 1, pp. 53-58, 2002.

[18] Q. Guo, S. Guo, and Z. Wang, "A type of esophageal stent coating composed of one 5-fluorouracil-containing EVA layer and one drug-free protective layer: in vitro release, permeation and mechanical properties," Journal of Controlled Release, vol. 118, no. 3, pp. 318-324, 2007.

[19] Y. Yuan, C. Liu, and M. Yin, "Plasma polymerized n-butyl methacrylate coating with potential for re-endothelialization of intravascular stent devices," Journal of Materials Science: Materials in Medicine, vol. 19, no. 5, pp. 2187-2196, 2008.

[20] H. Wang, Y. Liu, Y. Yang, T. Deng, G. Shen, and R. Yu, "A protein A-based orientation-controlled immobilization strategy for antibodies using nanometer-sized gold particles and plasmapolymerized film," Analytical Biochemistry, vol. 324, no. 2, pp. 219-226, 2004.

[21] Y. B. J. Aldenhoff, R. Blezer, T. Lindhout, and L. H. Koole, "Photo-immobilization of dipyridamole (Persantin) at the surface of polyurethane biomaterials: reduction of in vitro thrombogenicity, Biomaterials, vol. 18, no. 2, pp. 167-172, 1997.

[22] Y. Bai, C. G. Koh, M. Boreman et al., "Surface modification for enhancing antibody binding on polymer-based microfluidic device for enzyme-linked immunosorbent assay," Langmuir, vol. 22, no. 22, pp. 9458-9467, 2006. 

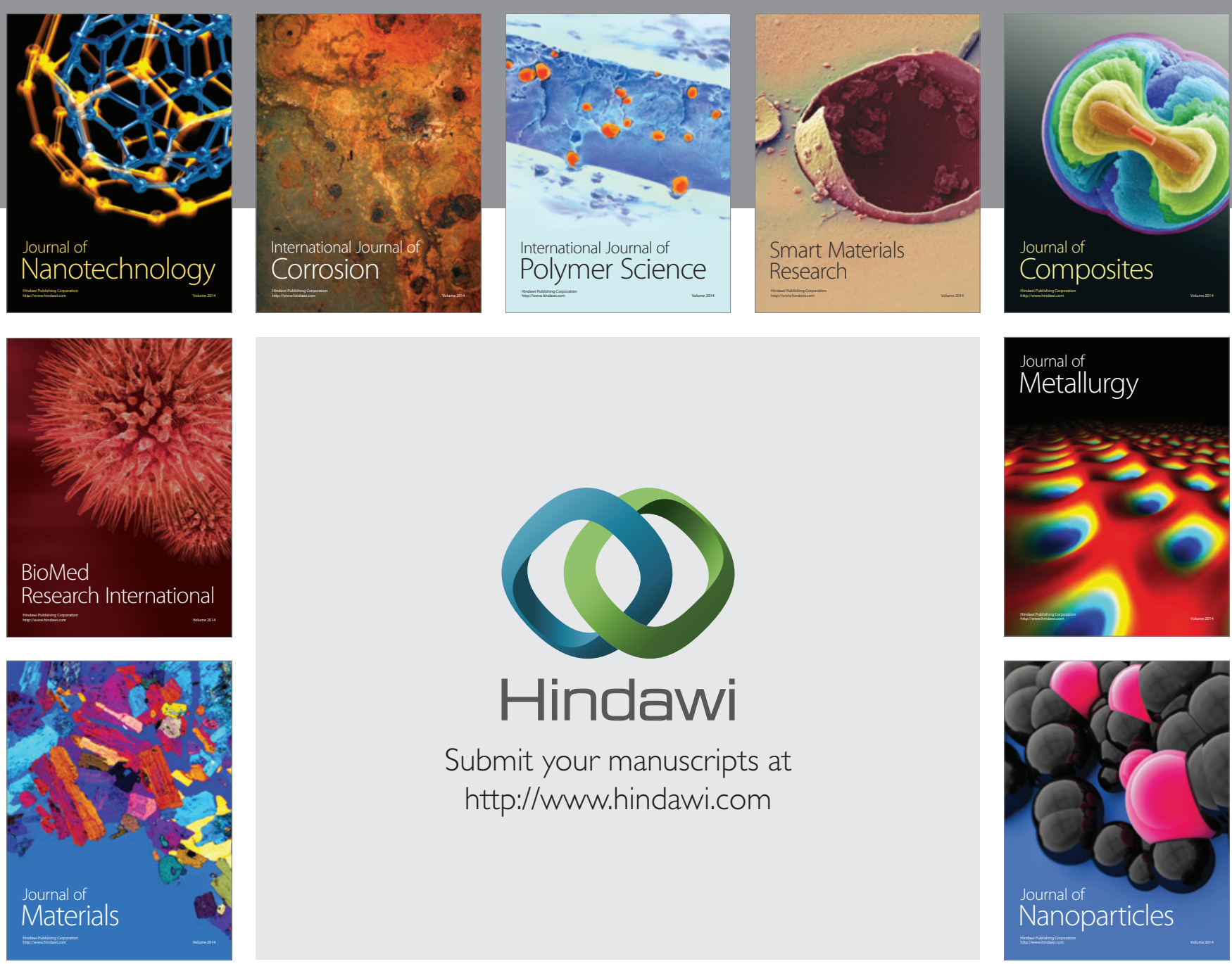

Submit your manuscripts at http://www.hindawi.com
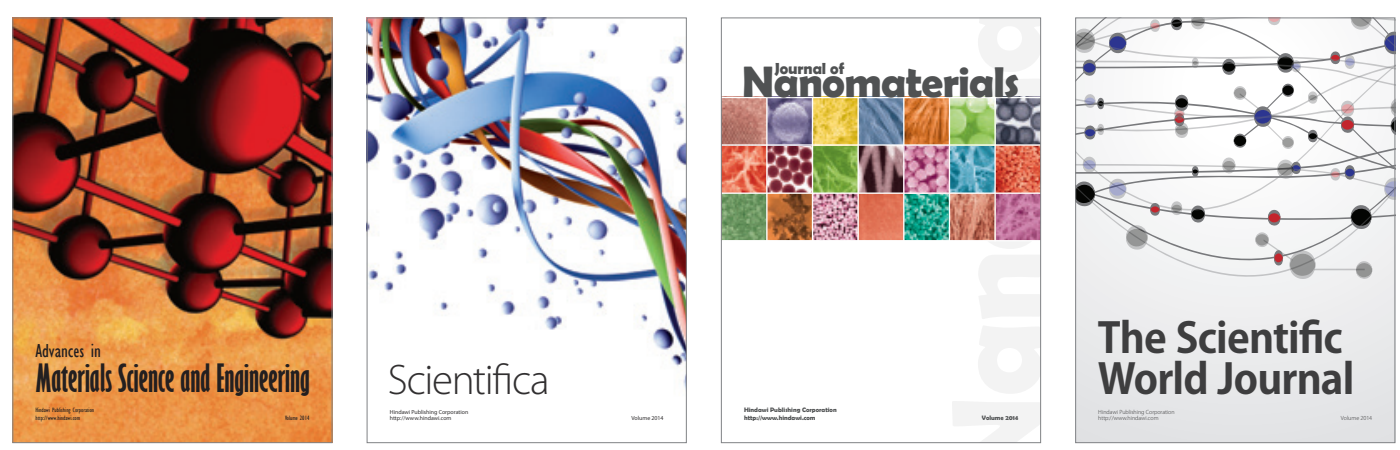

\section{The Scientific World Journal}
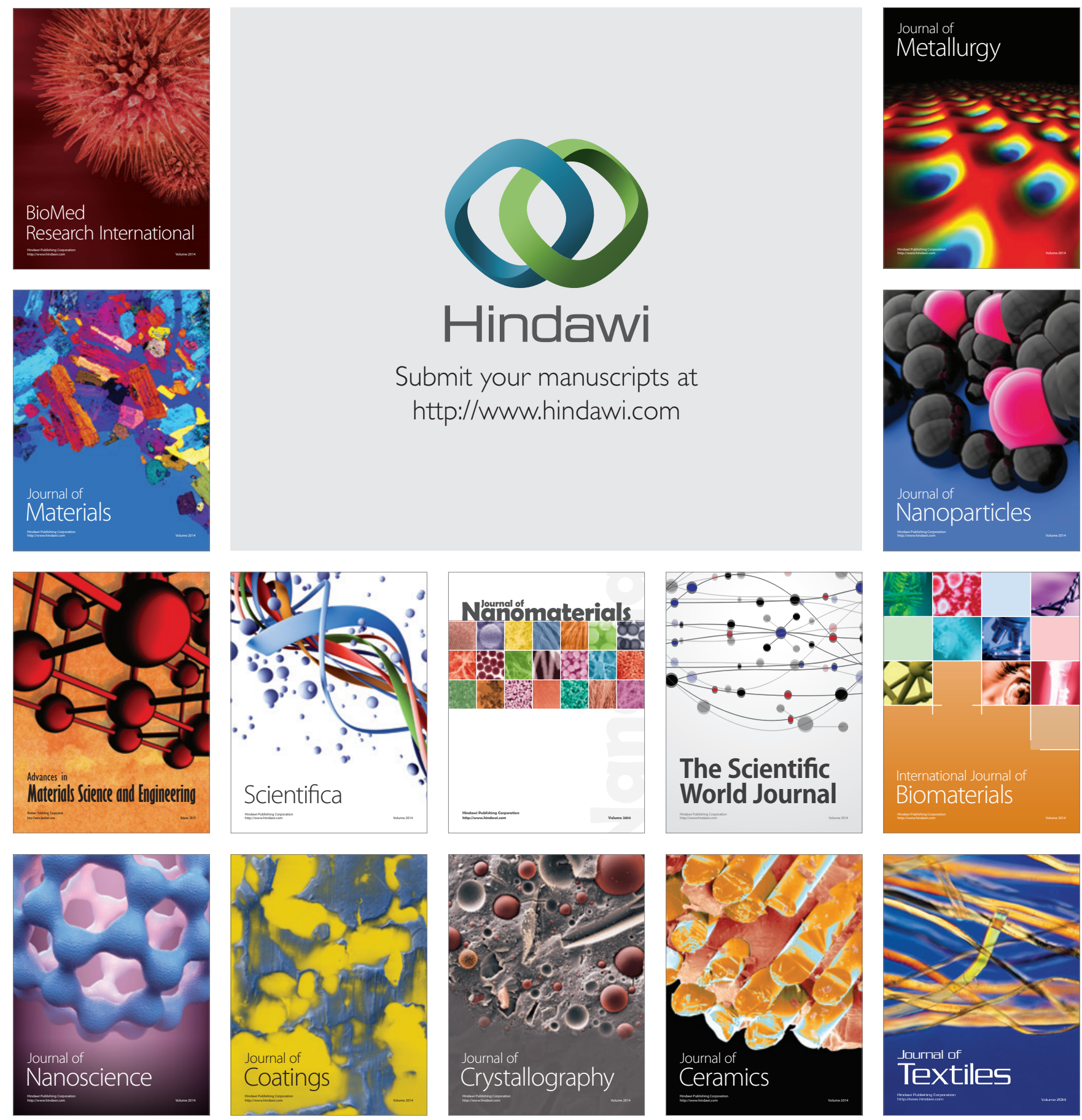